\title{
«Bolsa del Diablo/Bolsa de Color» PROYECTO DE VINCULACIÓN \\ BARRIO-UNIVERSIDAD-GOBIERNO, PARA LA \\ MEJORA DEL ESPACIO PÚBLICO EN LA ZONA \\ de Monumentos de la Ciudad \\ de Puebla, calle 24 Poniente
}

«Bolsa del Diablo/Bolsa de Color» Linkage Project

Neighborhood-university-government, for the Improvement of Public

Space in the Monuments Zone of the Puebla City, 24th West Street

\author{
Adriana Hernández Sánchez \\ adna0909@hotmail.com \\ Benemérita Universidad Autónoma de Puebla \\ Christian Enrique de la Torre Sánchez \\ christian.e.delatorre@gmail.com \\ Benemérita Universidad Autónoma de Puebla \\ Bernardo Aco Castañeda \\ nardok_200891@hotmail.com \\ Universitat de Barcelona \\ César Javier Rojas Salgado \\ rojas.salgado@hotmail.com \\ Universitat de Barcelona
}

RESUMEN: «Bolsa de Color» tuvo como objetivo una actuación puntual (aplicación de color en fachada) en la calle 24 Poniente del Barrio del Refugio, Puebla, México. Desde el siglo xvi, «La Bolsa del Diablo» es una calle que destaca por una forma irregular diferente al resto de las manzanas del Centro Histórico, lo que impide la visibilidad desde las esquinas. Hasta el xix existían hornos de cal y las vecindades han albergado una gran cantidad de habitantes, entre ellos grupos conflictivos. Antes de la intervención, el patrimonio construido estaba muy deteriorado y existía poca permeabilidad, ya que el imaginario negativo la había mantenido ajena a cualquier intervención. 
Es una calle donde hay poco tráfico vehicular y donde es común encontrar a los niños jugando al fútbol y a las canicas.

Con el uso de color en las fachadas y el trabajo voluntario de vecinos, universitarios y el Ayuntamiento de Puebla, se inició un proyecto de apropiación y valoración de los espacios públicos a partir de diversas actividades, que van desde las recreativas y culturales, hasta talleres y proyectos para la mejora del barrio.

Este trabajo es producto de la línea de investigación «Espacio Público, Participación Ciudadana y Centro Histórico» de la Facultad de Arquitectura de la Benemérita Universidad Autónoma de Puebla y del grupo Re Genera Espacio.

Palabras clave: participación ciudadana, calle, color, espacio público, justicia espacial.

ABSTRACT: Bolsa de Color was planned as a one-off performance (application of color on façade) on the street of 24 Poniente in the El Refugio neighbourhood of Puebla, Mexico. Since the sixteenth century, the irregular shapes of the street of La Bolsa del Diablo, which prevent visibility from the corners, have set it apart from the rest of the old city centre. Until the nineteenth century, lime kilns were in operation and the vecindades (collective housing) were home to many inhabitants, including conflicting groups.

Before the Bolsa de Color, the street's buildings were in a serious state of deterioration and there was little movement either in or out, its negative image deterring any intervention. The street has little traffic and children can often be seen playing soccer or marbles.

Using color on the façades and with voluntary help from neighbors, university students and the Puebla city government, a project was begun to recover and promote public spaces. The initiative embraced a range of recreational and cultural activities, including workshops and projects to improve the neighborhood. 
The present paper is the result of the research project Public Space, Citizen Participation and Historical Center at the Architecture Department of the Benemérita Universidad Autónoma de Puebla and the Re Genera Espacio group.

KEYWORDs: citizen participation, street, color, public space, spatial justice.

RESUM: «Borsa de Color» va tenir com a objectiu una actuació puntual (aplicació de color en façana) al carrer 24 Ponent del Barri del Refugio, Puebla, Mèxic. Des del segle xvi, «La Borsa del Diable» és un carrer que destaca per una forma irregular diferent a la resta de les illes del Centre Històric, el que impedeix la visibilitat des de les cantonades. Fins al xix existien forns de calç i el veïnat ha albergat una gran quantitat d'habitants, entre ells grups conflictius. Abans de la intervenció, el patrimoni construït estava molt deteriorat i existia poca permeabilitat, ja que l'imaginari negatiu l'havia mantingut aliè a qualsevol intervenció. És un carrer on hi ha poc trànsit vehicular i on és comú trobar als nens jugant a futbol i a les boles.

Amb l'ús de color a les façanes i el treball voluntari de veïns, universitaris i l'Ajuntament de Puebla, es va iniciar un projecte d'apropiació i valoració dels espais públics a partir de diverses activitats, que van des de les recreatives $\mathrm{i}$ culturals, fins a tallers i projectes per a la millora del barri.

Aquest treball és producte de la línia d’investigació «Espai Públic, Participació Ciutadana i Centre Històric» de la Facultat d'Arquitectura de la Benemérita Universidad Autónoma de Puebla i del grup Re Genera Espacio.

Paraules clau: participació ciutadana, carrer, color, espai públic, justícia espacial. 


\section{Justicia espacial y el derecho a la ciudad en un mundo global}

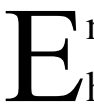

n el contexto actual donde la globalización ha cobrado gran importancia, ha surgido esta constante lucha de las ciudades por permanecer en el mapa global creando políticas de promoción y marketing que exportan una imagen que les proporcione lugar en él. La pregunta clave es: ¿A quién y qué representa esta imagen?

A través de las distintas intervenciones urbanas realizadas en los últimos años en la ciudad de Puebla, México, es fácil observar el crecimiento de la desigualdad espacial. Se favorece la creación de nuevos espacios públicos y la construcción de «obras emblemáticas» para la ciudadanía, con el fiel trasfondo de que van encaminadas a fortalecer la imagen de la «ciudad moderna» y la atracción turística. Estas políticas incrementan la polarización de los espacios públicos al no generar un desarrollo armonioso entre los nuevos proyectos y los ya construidos. Siendo estos últimos los espacios de la realidad donde se desarrolla la ciudadanía.

Por un lado, se busca crear una imagen para «vender mejor» la ciudad, para promocionarla. Mientras que por el otro (el de la ciudadanía) se vive un abandono de los barrios y colonias populares, el urbanismo presenta grandes déficits y genera la exclusión de amplios sectores de la ciudad.

Ya lo señala Nuria Benach (1993), como muchos otros autores, la ciudad es vista como un producto a colocarse en el mercado.

Ante este panorama de contraposición entre los intereses ciudadanos y las políticas urbanas, subrayamos el papel de los diseñadores, planificadores urbanos y organizaciones civiles como agentes de cambio que buscan mediar ambos intereses con la intención de contribuir a la construcción de la ciudad desde una perspectiva más justa e incluyente para todos.

El barrio del Refugio pertenece al Centro Histórico de la ciudad de Puebla, pero al encontrarse en su periferia no tiene los mismos privilegios de las políticas de intervención por parte de la administración pública. Es decir, se siguen centralizando las intervenciones de mejora urbana en las manzanas más rentables que establece el sector turístico de la ciudad, dejando el resto del espacio en condiciones de degradación física y también social. 
¿Cómo aminoramos esta desigualdad urbana? ¿Dónde queda el derecho de la población de estos sectores a gozar de un espacio digno y de calidad? ¿Cómo hacerlos partícipes en la construcción de la ciudad? Éstas, y muchas cuestiones más, son algunos de los puntos que tratamos de resolver y ejemplificar a través de la ejecución del proyecto de intervención Bolsa de Color en el barrio del Refugio. Realzando la importancia de las actuaciones locales, donde la ciudadanía se convierte en el agente principal de cambio con el apoyo de otros actores, pero que, sin duda alguna, garantizan el goce pleno de su derecho a la ciudad y a la contribución de la justicia espacial.

\section{El qué. Antecedentes históricos y situación previa al proyecto}

La Bolsa del Diablo es el nombre con el que se conoce a la calle 24 Poniente, entre 5 y 7 Norte, en el Barrio del Refugio (ver il. 1) y es parte de la Zona de Monumentos Históricos de Puebla. Se trata de una de las calles que en el imaginario colectivo es identificada como una de las más peligrosas de la ciudad, ya que se dice que en diversos periodos ha sido guarida de bandas delictivas a partir de la segunda mitad del siglo XIX.

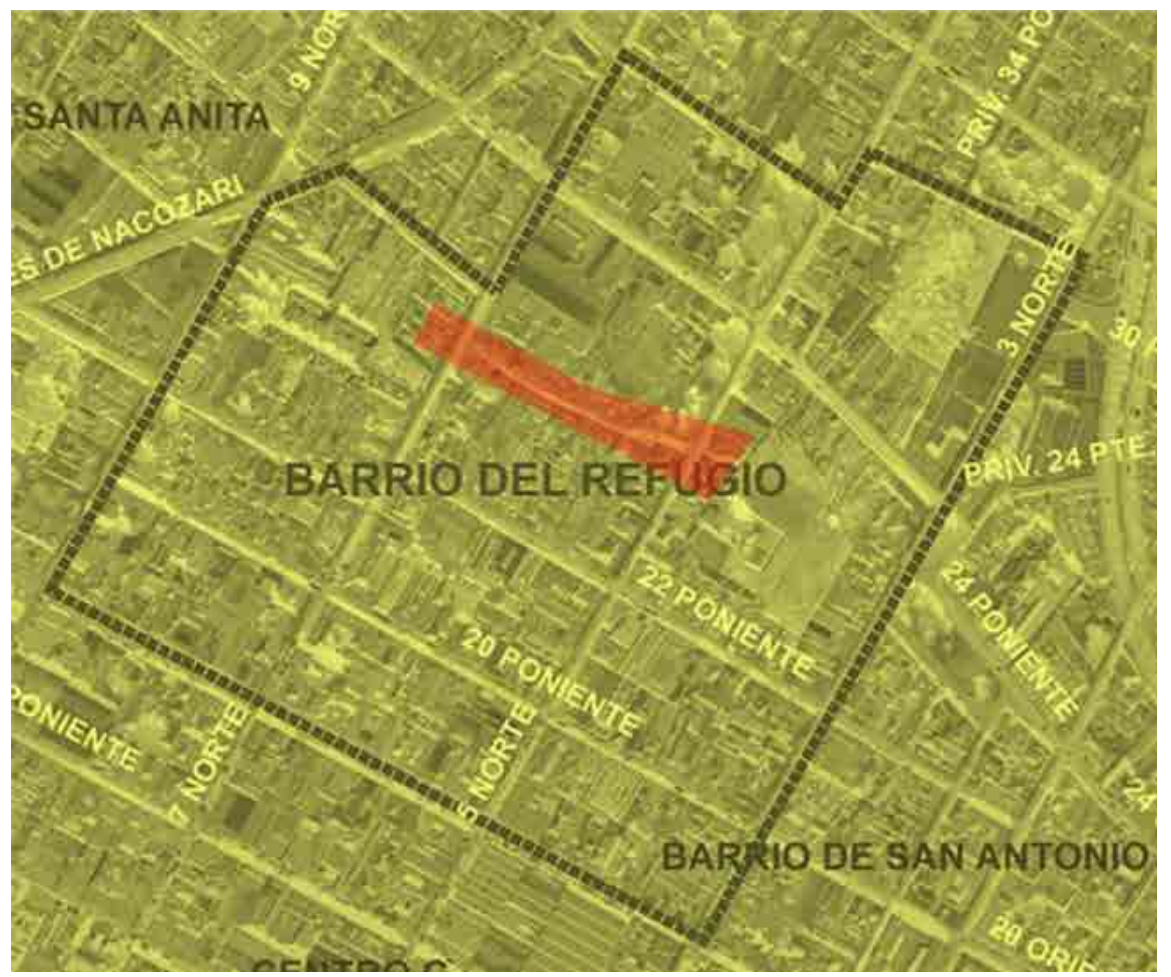

Ilustración 1. Calle Bolsa del Diablo en el barrio del Refugio de la ciudad de Puebla (Imagen editada a partir de Google Maps). 
Tomando como referencia el libro Las calles de Puebla de Hugo Leicht (1967), se elabora esta cronología con los siguientes datos históricos de la calle:

Tabla 1.

Cronología con datos históricos de la avenida 24 Poniente 500, antigua de Hornos o «La Bolsa del Diablo», según el libro «Las calles de Puebla» (Leicht, 1967, págs. 189-190).

\begin{tabular}{|c|c|}
\hline Añ๋ & Acontecimiento \\
\hline 1612 & $\begin{array}{l}\text { En los Libros del Cabezón se menciona que los hornos de cal sirven para señalar el } \\
\text { extremo norte de las actuales calles } 5 \text { y } 7 \text { Norte-Sur. }\end{array}$ \\
\hline 1698 & $\begin{array}{l}\text { En el plano de la ciudad de Puebla elaborado por Cristophorus de Guadalaxara, se } \\
\text { aprecia un trazo irregular de la calle, distinto al de las manzanas del casco central } \\
\text { español. }\end{array}$ \\
\hline 1742 & El barrio es conocido como el «Real de las Caleras» y luego del Refugio. \\
\hline 1754 & $\begin{array}{l}\text { En el plano de la ciudad de Puebla elaborado por Joseph de Medina, hay tres caleras } \\
\text { en la manzana situada al norte de la } 22 \text { Poniente } 500 .\end{array}$ \\
\hline 1773 & En el padrón municipal se cita la existencia de seis hornos de cal. \\
\hline 1807 & $\begin{array}{l}\text { La manzana norte tiene una huerta que contiene en su parte occidental tres hor- } \\
\text { nos. En cada una de las esquinas nordeste y sudeste hay un «cuadrito con edificios» } \\
\text { y en el ángulo sudoeste del último, lindando con esta avenida, está marcado un } \\
\text { cuarto horno. En un plano del Ayuntamiento se conoce como Calle de las Caleras. }\end{array}$ \\
\hline 1824 & $\begin{array}{l}\text { Se citan «la plazueleta que sube para el horno de Chávez» (sic) y la «callejuela que } \\
\text { entra por el mismo horno». }\end{array}$ \\
\hline 1852 & $\begin{array}{l}\text { La cuadra se titula «Calle del Horno de Chávez» en el directorio de la Guía de } \\
\text { ese año. }\end{array}$ \\
\hline $1856-1883$ & $\begin{array}{l}\text { La calle se conoce como Calle de los Hornos en los planos elaborados por Luis G. } \\
\text { Careaga y Sáenz. }\end{array}$ \\
\hline 1888 & $\begin{array}{l}\text { «Frente al Horno de Chávez, en la esquina de la calle } 7 \text { Norte } 2200 \text {, los planos de } \\
\text { Careaga marcan un edificio que pertenecía a la posesión de Ramírez, pero estaba } \\
\text { separado de sus caleras por parte de la huerta. En un tiempo había servido para } \\
\text { una locería, en que se fabricaba loza corriente; después lo habitaban peones de } \\
\text { los hornos, en parte gente de mala reputación, por lo cual lo bautizaron con el } \\
\text { nombre de La Bolsa del Diablo. Como “la casa conocida por La Locería” la citan en } \\
\text { el Registro Público de ese año. (Según la tradición oral, había sido un escondrijo de } \\
\text { contrabandistas, que allí ocultaban el pulque y otras mercancías que introducían sin } \\
\text { pagar los derechos en las garitas.)» (Leicht, 1967, pág. 190) }\end{array}$ \\
\hline 1890 & «La casa de la locería» es conocida también como «de la Bolsa del Diablo». \\
\hline 1902 & La cuadra se denomina Calle de la Bolsa del Diablo. \\
\hline $1905-1908$ & La calle se conoce como Calle del Horno de Chávez en el Registro Público. \\
\hline I91 I & $\begin{array}{l}\text { En los planos de Márquez y de Vidaurri, y en Nieto se usan los nombres: Locería } \\
\text { Vieja y Hornos. }\end{array}$ \\
\hline 1913 & Se conoce como Callejón de Chávez. \\
\hline 1915 & $\begin{array}{l}\text { En el plano de Soto, elaborado a solicitud de la Empresa de Anuncios Prácticos, se } \\
\text { lee como La Bolsa. }\end{array}$ \\
\hline 1916 & «La casa de la locería» es conocida como «La Bolsa del Diablo». \\
\hline 1925 & La calle se denomina como Calle de la Bolsa del Diablo. \\
\hline 1937 & En la fotografía aérea de la ciudad de Puebla se distingue la forma actual de la calle. \\
\hline
\end{tabular}


La forma irregular de la calle es una característica documentada en los planos antiguos de la ciudad de Puebla, como el de Cristophorus de Guadalaxara elaborado en 1698, y más evidenciado en los elaborados a partir del siglo XIX, como el de Luis G. Careaga y Sáenz de 1883 (ver il. $2)$. Es probable que esta situación en la traza, con nula visibilidad de un extremo de la calle al otro, influya en la percepción de inseguridad de la misma.

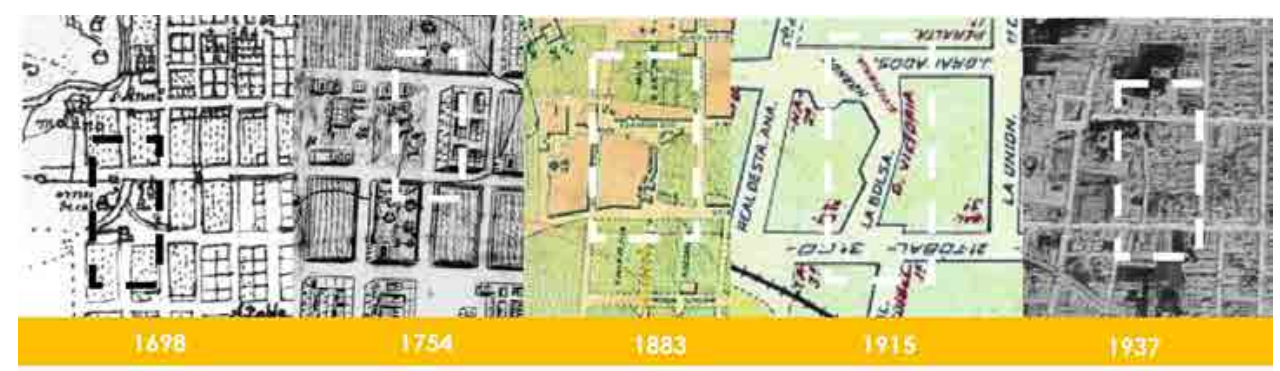

Ilustración 2. Fragmentos de planos antiguos de la ciudad de Puebla donde se aprecia la calle 24 Poniente 500, antigua de Hornos o «La Bolsa del Diablo»: 1698 plano elaborado por Cristophorus de Guadalaxara, 1754 plano elaborado por Joseph de Medina, 1883 plano elaborado por Luis G. Careaga y Sáenz, 1915 plano elaborado a solicitud de la Empresa de Anuncios Prácticos y 1937 plano aéreo de la ciudad de Puebla (Vélez Pliego, Guzmán Álvarez, \& et al, 1997).

En la actualidad, aún se conserva el muro de la fachada de la antigua casa de «La Locería» o de la «Bolsa del Diablo», que como menciona Hugo Leicht (1967), y atendiendo a la tradición oral, sirvió para la realización de actividades ilícitas como el contrabando y el escondite para ladrones. Según los testimonios de algunos vecinos, hasta hace unos «veinte o treinta años», el inmueble era una gran vecindad que fue derrumbándose con el paso del tiempo, primero con el sismo de 1973 y luego con el de 1985, hasta quedar únicamente un lote baldío con una fachada que tiene los vanos tapiados. Es a partir del análisis del libro de Leicht y de los planos antiguos de la ciudad de Puebla, como el de Luis G. Careaga y Sáenz de 1883 (Vélez Pliego, Guzmán Álvarez, \& et al, 1997), que puede definirse que se trata de dicha casa (ver il. 4 y 5 ). 


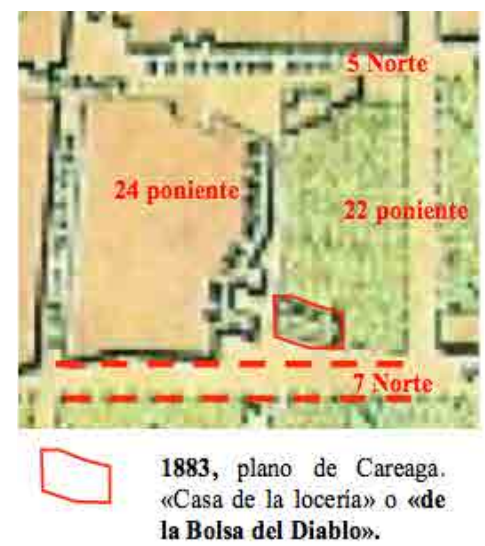

Ilustración 3. Fragmento del plano de la ciudad de Puebla elaborado por Luis Careaga y Saénz en 1883, donde se identifica la antigua casa de «La Locería» o de «La Bolsa del Diablo» (Vélez Pliego, Guzmán Álvarez, \& et al, 1997).

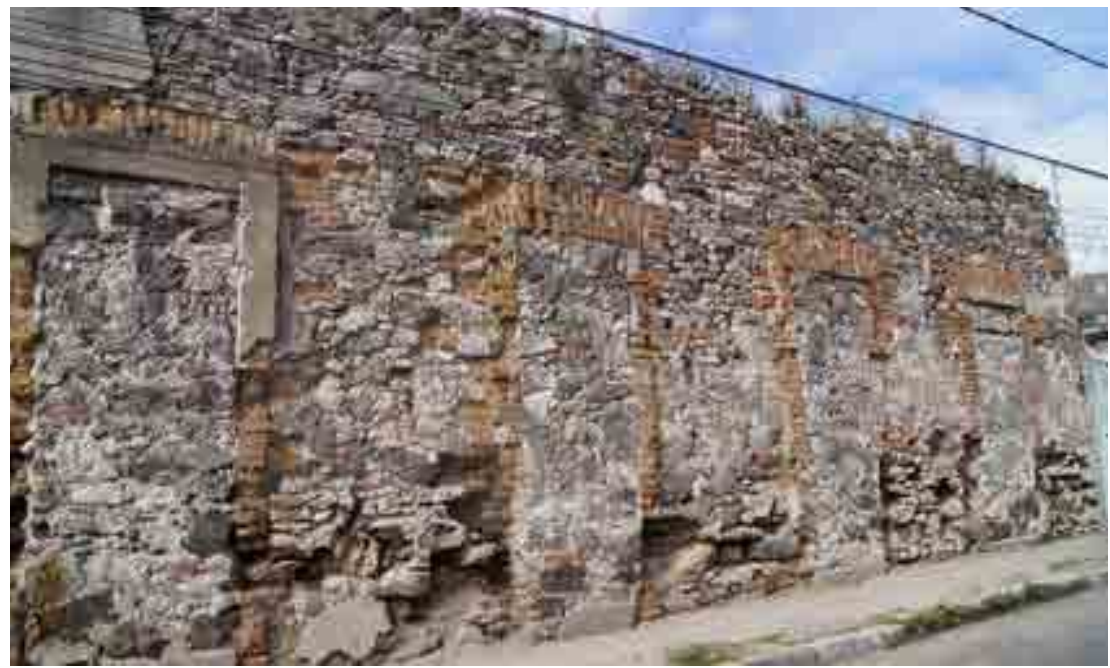

Ilustración 4. Fachada de la antigua casa de «La Locería» o «La Bolsa del Diablo», 24 Poniente 519 o 7 Norte 2208, en el Barrio del Refugio, año 2013. (Fotografía de Adriana Hernández Sánchez.)

La propia historia de este sector ha favorecido a su continua estigmatización no solamente por parte de la población externa al barrio sino también de las personas que lo habitan, quienes señalan esta calle como un «lugar conflictivo y peligroso». 
Además, si añadimos las condiciones físicas del lugar se fortalece la construcción de esa imagen negativa. Ante esto, consideramos importante destacar (previo a la intervención) la decadencia del espacio público, la infraestructura y la vivienda (ver il. 5). Visualmente era una calle poco atractiva por el grave deterioro del plano horizontal y vertical (aceras, carril y fachadas) y concentraba grandes cantidades de desechos y grafitis. La falta de iluminación pública era otro factor que por la noche generaba inseguridad y la hacía aún más impermeable. A pesar de que uno de sus límites conecta con uno de los dos parques del barrio (con el Ángela Peralta) no lograba articularse con él.

Aunque muchos de los inmuebles se encuentran vacíos, cabe destacar el gran número de población que ahí habita, debido a la presencia de vecindades, un sistema de vivienda colectiva. No obstante, sus condiciones físicas no eran aptas para generar una mejor calidad de vida.

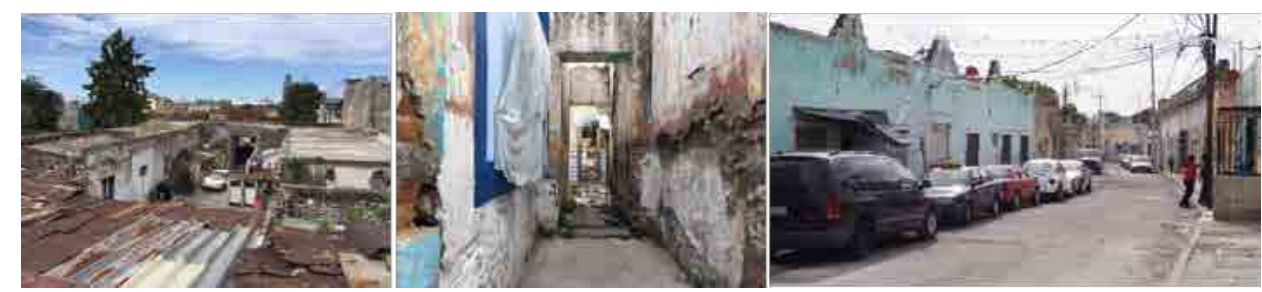

Ilustración 5. Estado físico del espacio público e inmuebles en la Bolsa del Diablo. (Fotografía de Adriana Hernández Sánchez.)

Es importante señalar la problemática social que también es consecuencia de los problemas físicos del lugar. La desconfianza de la población hacia el sistema político generada por las nulas actuaciones de mejora del barrio, propicia que no exista interés por participar en temas del desarrollo de la ciudad, de su contexto. La desarticulación crea una división interna que no ayuda a la organización vecinal. La misma estigmatización de los vecinos de otras calles no es solamente hacia el espacio físico de la Bolsa del Diablo, es también hacia la población que ahí habita. Cabe destacar que el sistema de vivienda colectiva se asocia a la población de escasos recursos. Lo anterior hace de este sector un lugar vulnerable física, económica y socialmente, desarticulado del resto de la ciudad. 


\section{¿Quiénes participan? Los actores: vinculación universitaria, so- ciedad, Ayuntamiento}

La participación social en los proyectos de investigación contribuye a relacionar la investigación con la gestión. En este caso concreto se trata de ver a la arquitectura y al diseño urbano desde una visión no sólo técnica, también más humana, de incidir en zonas deterioradas por medio de actuaciones como la propuesta de la línea de investigación Espacio Público, Participación Ciudadana y Centro Histórico, promovida por la Benemérita Universidad Autónoma de Puebla.

Desde el 2012, con esta línea se han desarrollado proyectos de tipo urbano y arquitectónico que impactan de una manera importante en zonas deterioradas dentro del Centro Histórico de la ciudad de Puebla, incorporando a estudiantes de arquitectura, urbanismo, ciencias políticas, comunicación y conservación del patrimonio. Se han abordado diferentes iniciativas como el impulso de una biblioteca en el barrio de San Antonio; la donación de plantas y árboles por parte del área de parques y jardines del Ayuntamiento al barrio del Refugio; la elaboración de un proyecto ejecutivo del parque de Santa Anita con un monto de más de cuatro millones de pesos; la obtención de recursos para la elaboración de un libro ante el Fondo Nacional para la Cultura y las Artes (FONCA) sobre la historia del barrio de San Antonio; y en el 2014 el proyecto denominado Bolsa de Color en el barrio del Refugio, donde se pintaron las fachadas de la calle 24 Poniente, conocida como Bolsa del Diablo. En el 2016, el grupo se establece en una vecindad del barrio y en el 2017 se llega a un acuerdo de comodato para la habilitación de un predio como un espacio público temporal, con la participación de la población y promoviendo la conservación del patrimonio.

Lo anterior incide en lugares donde no se estaba acostumbrado a trabajar con la comunidad, donde la población está inmersa en un proceso de inseguridad y desconocimiento de cómo acercarse a las instancias correspondientes, además de la apatía que propicia la fragmentación como sociedad o comunidad.

Ante esta situación, el papel de las organizaciones sociales y de los profesionales involucrados en el «hacer ciudad» cobra importancia como mediador 
y facilitador de procesos en este tipo de contextos, lo que es parte de nuestra responsabilidad social.

Este proyecto de imagen urbana y espacio público se encaminó a mejorar las condiciones de vida de los habitantes. De este modo, son ellos los actores base para la trasformación. Como señala la UnESCo (2016), «las ciudades centradas en las personas son espacios centrados en la cultura que respetan la diversidad cultural y garantizan la inclusión social y fomentan la creatividad y la innovación».

Las relaciones entre los actores involucrados en el proyecto Bolsa de Color se pueden observar en el siguiente cuadro:

\section{Tabla 2.}

Mapa de actores del proyecto Bolsa de Color. La línea de investigación Espacio Público, Participación Ciudadana y Centro Histórico es el origen del proyecto, del cual se derivan dos ejes: el social (izquierda) y el físico (derecha), que a su vez cuentan con participantes directos (línea continua) e indirectos (línea punteada).

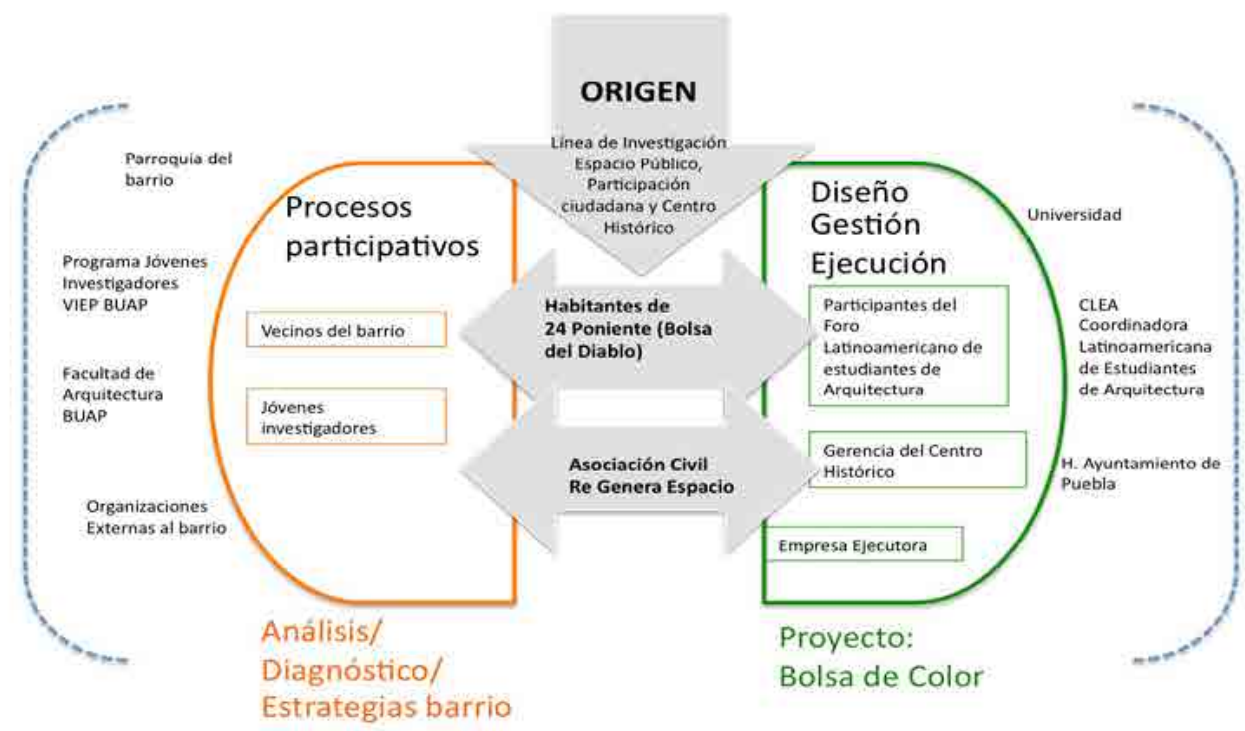


En relación a la tabla 2, de las dinámicas establecidas entre los actores para la ejecución del proyecto, resaltamos lo siguientes puntos:

a) Los habitantes de la 24 Poniente son partícipes en todas las etapas del proyecto. Desde el diseño, la gestión y ejecución, acompañados en todo momento por la organización social.

b) La iglesia del barrio, como actor clave para el acercamiento a la población.

c) La importancia de que actores externos (personas $u$ organizaciones) se impliquen a lo largo del proceso aportando nuevas ideas y ayudando a través de diversas actividades para generar la apropiación del espacio y disminuir la estigmatización del lugar y sus habitantes.

d) La organización social como mediadora entre la población y la administración pública. Lo que incentiva a la población hacia un modelo de cambio a través de la gestión ante la administración.

e) La administración pública como elemento clave en la gestión de los recursos y como mediador entre las empresas ejecutoras (sector privado) y la organización civil.

f) La universidad como facilitadora para la integración de estudiantes a la ejecución de proyectos sociales.

g) La movilidad estudiantil internacional reflejada en el involucramiento de estudiantes de catorce países de Latinoamérica, gracias al vínculo entre la universidad y la organización civil Re Genera Espacio.

\section{El cómo. Proyecto de actuación Bolsa de Color}

Bolsa de Color surge a raíz del proyecto integral para el barrio del Refugio que se ha trabajado desde el año 2013, en el cual se han realizado diversas acciones que van desde proyectos de involucramiento ciudadano, como el diseño participativo donde se han realizado talleres con la población para proyectos urbanos y arquitectónicos, hasta actividades de tipo cultural que han fomentado otras propuestas creativas dentro del barrio. 
La Bolsa del Diablo se caracteriza por la presencia de jóvenes y niños que en diferentes horas del día se apropian de la calle, ya sea jugando al fútbol, a las canicas o platicando en la banqueta.

La época de construcción de los inmuebles comprende de los siglos XVIII al xx, algunos han sido subdivididos y otros abandonados por los propietarios o sus herederos, lo que ha provocado, según testimonios de vecinos, la invasión de viviendas. En otros casos más afortunados se sigue conservando la vida en vecindad, factor que se puede destacar en la Bolsa del Diablo, ya que se practican actividades en conjunto y existe fraternidad entre vecinos y familiares.

El proyecto Bolsa de Color surge por la necesidad de establecer actuaciones puntuales en zonas deterioradas. El barrio ha estado al margen de todo tipo de intervención y sobre todo la calle 24 Poniente, que para el resto de la ciudad es identificada como una calle «poco deseada» y que la tradición la sigue marcando como demasiado peligrosa, lo que la había estigmatizado por mucho tiempo para que fuera atendida por algún programa gubernamental. (Ver il. 6.)

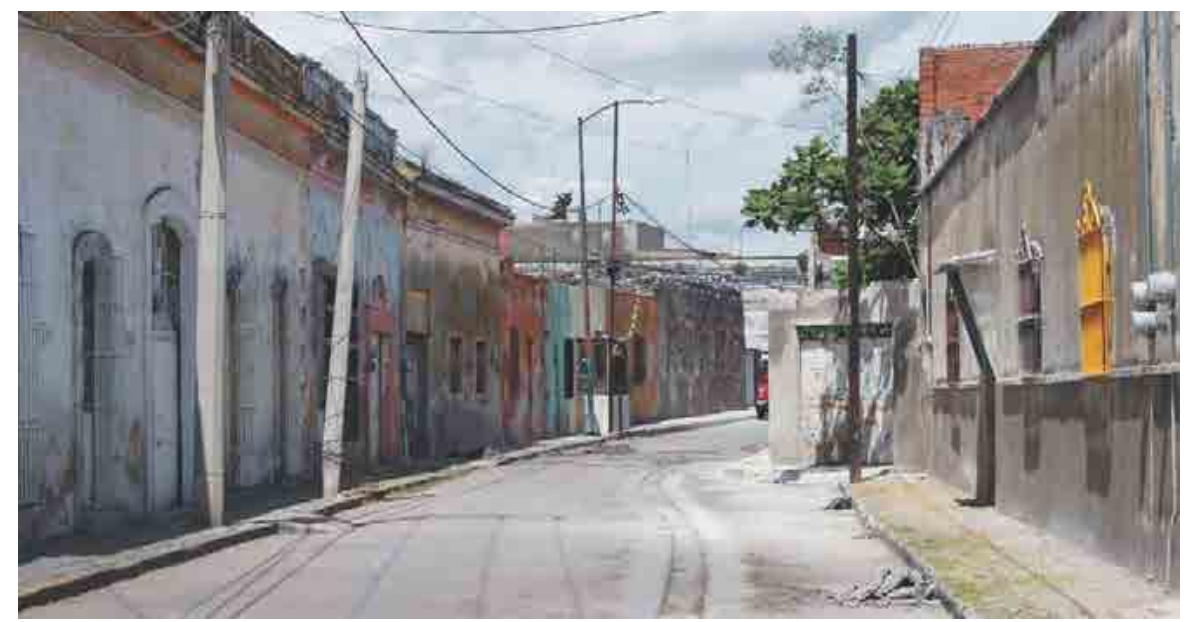

\section{Ilustración 6. Estado físico del espacio público e inmuebles en la Bolsa del Dia- blo antes de la intervención. (Fotografía de Adriana Hernández Sánchez.)}

La gestión del proyecto se ejecutó ante el Ayuntamiento de la ciudad de Puebla en el 2014. Días previos a estas jornadas, la población del barrio seleccionó los colores de sus fachadas a partir de la votación de dos propuestas 
probables para su aplicación y acordes a la normativa del Instituto Nacional de Antropología e Historia (INAH). Es decir, a cada vecino se le preguntó el color que le gustaba para sus muros y elementos de herrería como puertas y ventanas (ver il. 7). También los vecinos se organizaron para limpiar la calle, retiraron escombros y objetos, incluso chatarra y autos abandonados, que se habían acumulado a lo largo del tiempo en la vía pública. Mientras el Ayuntamiento, algunas semanas previas a la aplicación de pintura, realizó diversas obras de restauración en las fachadas de los inmuebles, debido a que algunas requerían un mantenimiento mayor (ver il. 8).

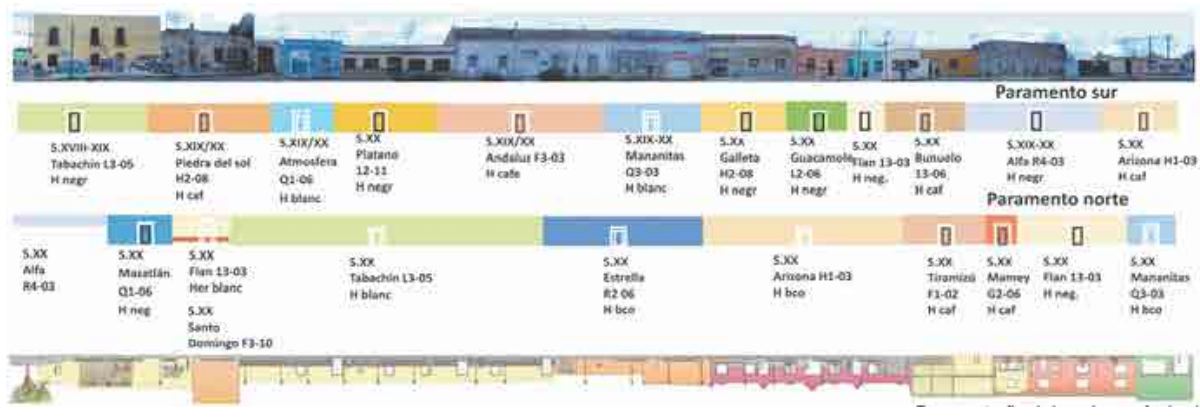

\footnotetext{
Ilustración 7. Propuesta final de color en fachadas, seleccionadas por los vecinos de la Bolsa del Diablo (Re Genera Espacio).
}
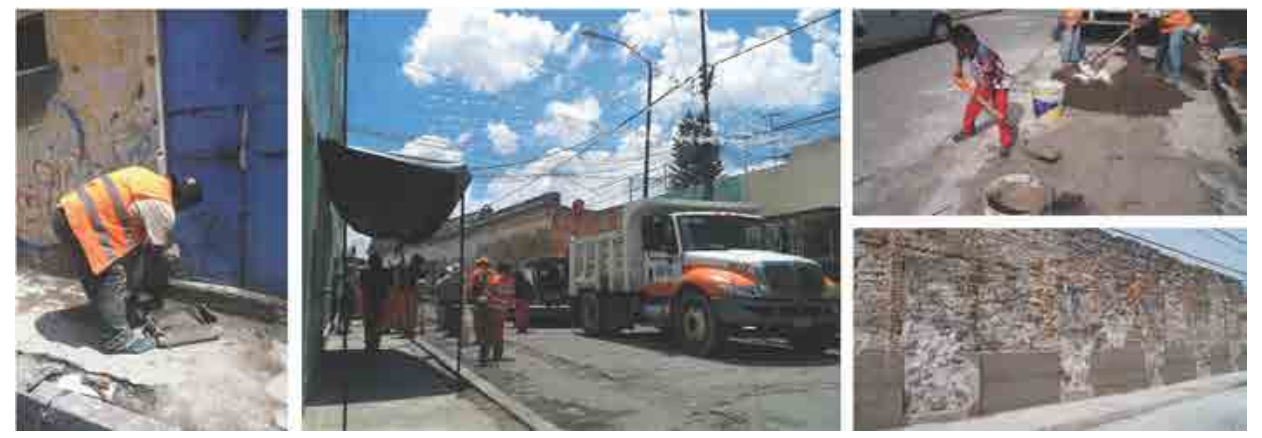

Ilustración 8. Primera etapa: restauración de fachadas de inmuebles por parte del Ayuntamiento y apoyo por parte de los niños.

(Fotografías de Adriana Hernández Sánchez.) 
En una de las etapas del proyecto destacó la presencia de jóvenes universitarios durante tres días consecutivos en el marco del II Foro Latinoamericano de Estudiantes de Arquitectura. Se trataba de universitarios provenientes de diferentes países, quienes a través de una capacitación contribuyeron con su mano de obra al raspado de las superficies y a prepararlas para la aplicación de pintura (ver il. 9).

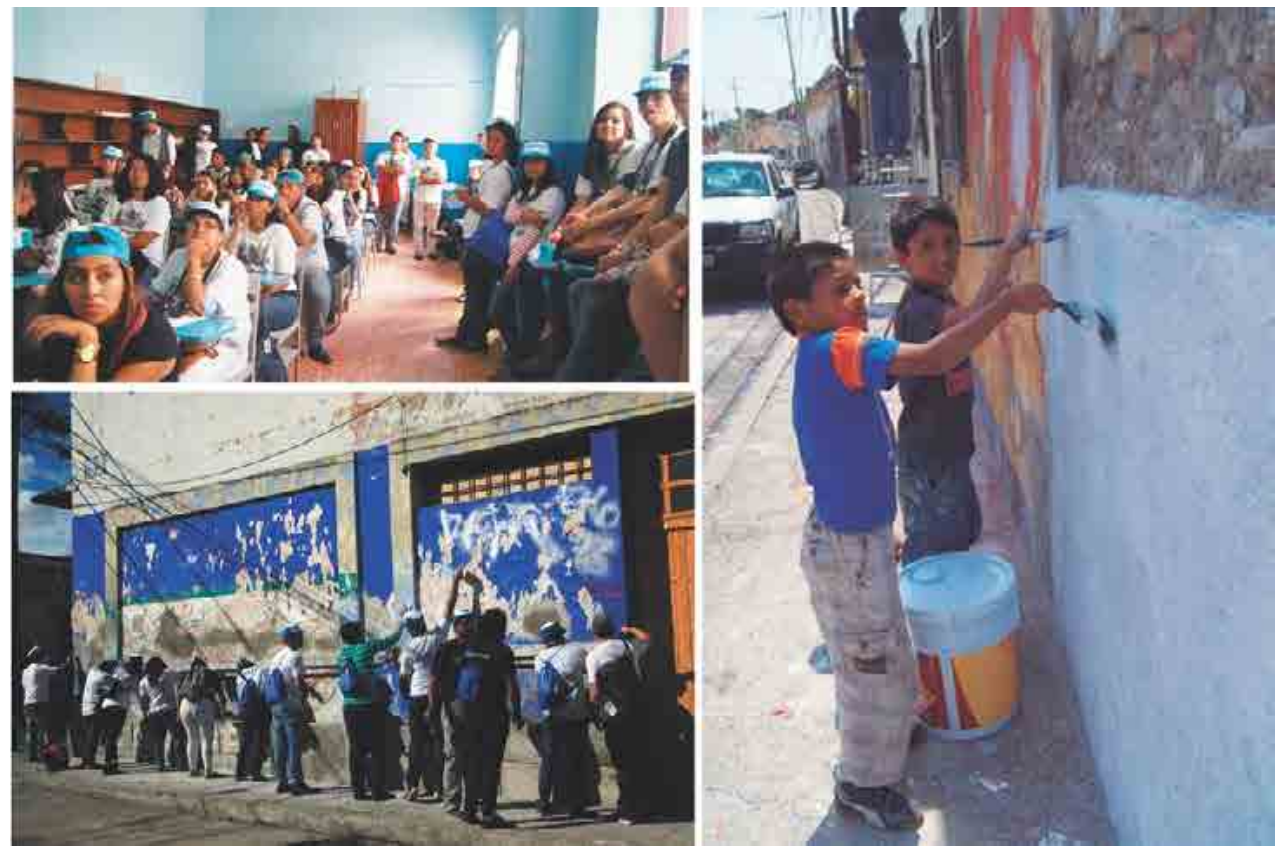

Ilustración 9. Segunda etapa: preparación de superficies y trabajo conjunto entre estudiantes y vecinos. (Fotografías de Adriana Hernández Sánchez.)

Posterior a la intervención de los jóvenes estudiantes de arquitectura asistentes al foro, la población continuó con las jornadas de mejoramiento de la imagen urbana los días sábados y domingos, en una actividad donde padres, tíos e hijos, así como el grupo interdisciplinar Re Genera Espacio (RGE), participaron en la aplicación de pintura; a su vez, a la calle y a los parques del barrio se le suministraron nuevas luminarias por parte del municipio. Esto propició que la calle ahora sea una de las más iluminadas conectándola con el resto de los espacios públicos del barrio. Incluso se constituyó un grupo de niños, vecinos de la Bolsa y calles colindantes, que tomó la iniciativa de continuar con la pintura de las fachadas a la par del trabajo que realizaban los empleados muni- 
cipales y voluntarios de RGE, lo que causó un impulso positivo para seguir adelante y motivar a sus padres a trabajar incluso por las noches (ver il. 10 y 11).

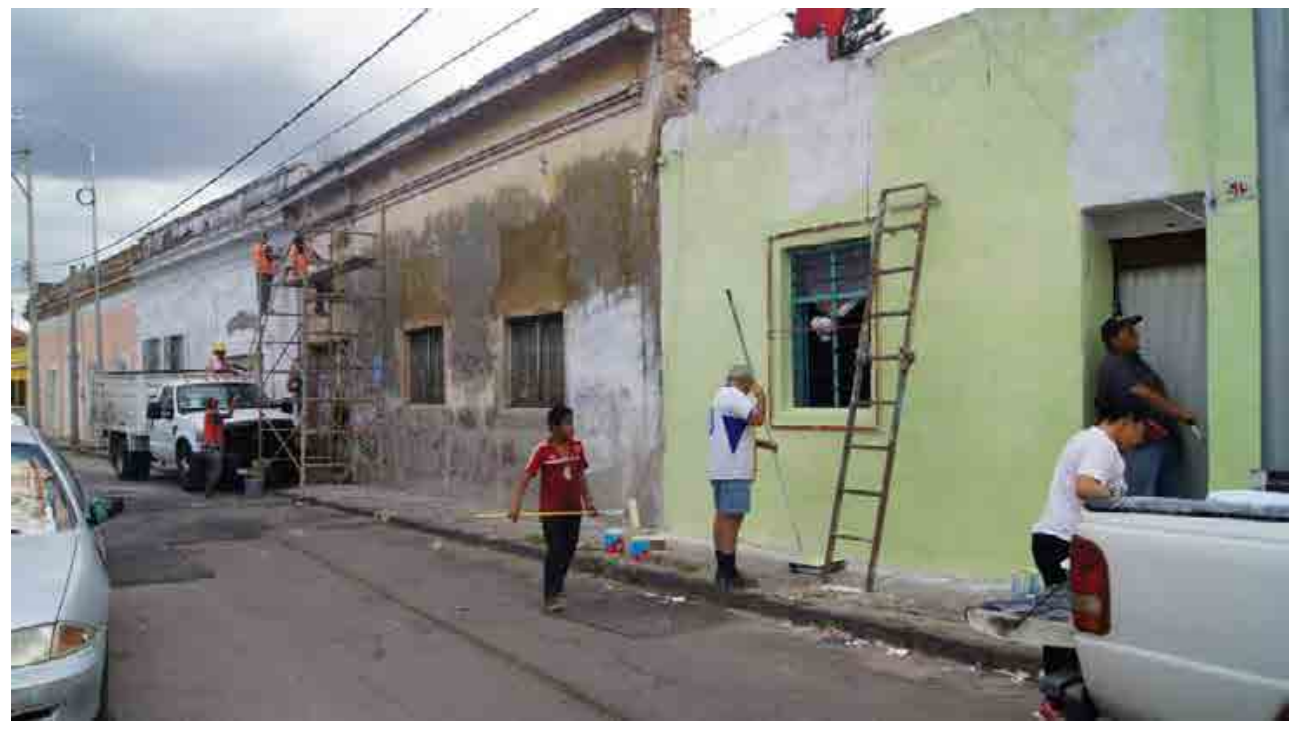

Ilustración 10. Trabajo conjunto entre vecinos, universitarios, voluntarios y personal del Ayuntamiento de Puebla para la pintura de fachadas.

(Fotografía de Adriana Hernández Sánchez.)

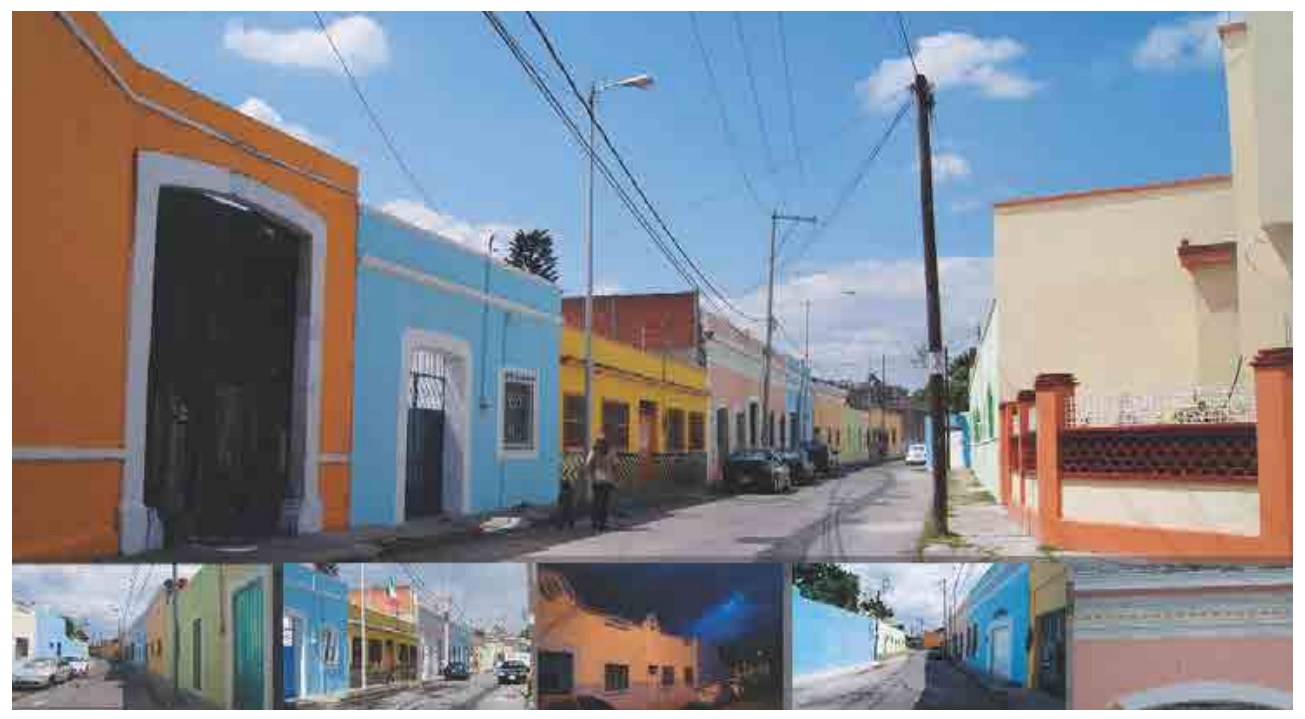

Ilustración 11. Resultado final. (Fotografías de Adriana Hernández Sánchez.) 


\section{Y todo esto... ¿Para qué?}

En primer lugar, muestra un proyecto que surge en un contexto donde las intervenciones van dirigidas principalmente a la promoción de la ciudad. Por tanto, su importancia radica en ir en contra de ello, colocando en primer plano a la ciudadanía.

La permanencia en la calle posibilitó descubrir el rostro de la misma, en donde se convive de una manera fraternal entre amigos, familiares y grupos externos, convirtiéndolo a la vez en un espacio de encuentro social y poniendo de manifiesto la necesidad de mejorarlo. Como mencionan Borja y Muxí (2000), «los espacios públicos requieren un debate público, la participación ciudadana, a lo largo del proceso de concepción, producción y gestión».

La esencia del proyecto no consistió solamente en la aplicación de pintura sobre las fachadas, sino que ha significado una nueva forma de ver y vivir el espacio público, tanto para los que ahí viven como para la población externa que se vio implicada. Fue un símbolo de demanda hacia la administración municipal para mirar a todos estos espacios que desde hace tiempo no son objeto para la intervención y mejora. Aquí la población se convirtió en un agente de cambio y de mejora en su entorno.

Es un proyecto que permite analizar el espacio público desde lo vivido, comprendiéndolo desde el uso que la gente le da, de su apropiación.

Además, a través de este proyecto se plantea que el patrimonio ya no sólo es responsabilidad de las instancias de gobierno de los tres niveles (federal, estatal y municipal), sino que cada vez es más convincente que la conservación del patrimonio también debe ser $r$ esponsabilidad de los habitantes, un proceso de apropiación e identidad, donde a través de la unión se pueden obtener buenos resultados.

El involucramiento de los vecinos como ejecutores de la obra es un factor para la apropiación y mantenimiento de este espacio público. Tres años después, la relación entre Re Genera Espacio y la comunidad se ha fortalecido. Sin embargo, ha sido difícil mantener la atención de la administración municipal hacia este sector. Pero ahora, a diferencia de años atrás, la población se encuentra más activa y confía en el cambio originado por ellos, buscando mejores condiciones de sus espacios desde las pequeñas acciones. 
Actualmente, el proyecto ejecutado Bolsa de Color es un parteaguas en la historia de la calle, ya que sus habitantes se muestran orgullosos de haber participado y ha propiciado una nueva forma de verse como comunidad que logra objetivos en conjunto a pesar de las diferencias que existen entre ellos.

Gracias a esta intervención, la calle es más permeable y propicia mayor vida social. Las nuevas luminarias permiten un mayor uso de la vía pública. Los vecinos y Re Genera Espacio continúan trabajando en diversas actividades que fomentan el buen uso del espacio público. Consideramos que lo más difícil de toda intervención urbana es la apropiación y, por tanto, hemos puesto énfasis en este punto para lograrlo. Muestra de ello, son los proyectos que ahora surgen desde la iniciativa de los vecinos y donde invitan a la organización a formar parte de ellos, como las jornadas de limpieza, el taller barrial de artes y oficios, los festejos como el día del niño o las posadas y este último año la habilitación de un espacio público temporal (la cancha ludoteca) en un terreno prestado por un propietario. Por lo anterior, es necesario que el grupo siga contribuyendo a la gestión de recursos para la calle y también para el resto del barrio. La Bolsa del Diablo se convierte en un elemento articulador entre los patios de las vecindades, los parques y los nuevos equipamientos como la cancha ludoteca.

La fortaleza de esta calle es la participación de la población. Al principio promovida por el grupo, pero que siempre ha estado vigente, ya que ellos han planteado a lo largo de todo el año diferentes actividades, organizan sus fiestas patronales, sus partidos de fútbol. Lo que en realidad ha venido a hacer el grupo es enseñarles otras formas de participación donde la arquitectura y el urbanismo estén presentes. El reto es la permanencia de este grupo social como uno de los ejemplos de la vida barrial que aún se conservan en el centro histórico, ya que están en riesgo de desparecer por situaciones externas, como las políticas de vivienda propuestas por las autoridades. En el caso del grupo, el reto está en seguir construyendo nuevas iniciativas con ellos. 


\section{Referencias}

BenaCh, N. (1993). Producción de imagen en la Barcelona del 92. Estudios Geográficos, LIV (212), 483-505. Obtenido de http://diposit.ub.edu/ dspace/handle/2445/63563

Borja, J. y Muxí, Z. (2000). El Espacio Público, Ciudad Y Ciudadanía. Barcelona. Recuperado de http://www.esdi-online.com/repositori/ public/dossiers/DIDAC_wdw7ydy1.pdf.

Contreras Cruz, C. (1986). La ciudad de Puebla estancamiento y modernidad de un perfil urbano en el siglo XIX (Primera ed.). Puebla, Puebla, México: Universidad Autónoma de Puebla.

Estrada Urroz, R. (2001). Espacios cuidados, segregados y prohibidos en la ciudad de Puebla en las tres primeras décadas del siglo xx. En M. Villadevall i Guasch, Ciudad, Patrimonio y Gestión (págs. 333-353). Puebla: Benemérita Universidad Autónoma de Puebla.

Gobierno del Estado de Puebla (1985). Catálogo de monumentos arquitectónicos, artísticos e históricos de la ciudad de Puebla. Puebla.

Hernández Sánchez, A. (2000). La vivienda en los barrios tradicionales de Puebla: El Alto, Analco, La Luz y Xanenetla (Tesis de Maestría). Universidad Nacional Autónoma de México.

- (2009). El Espacio Público en el Centro Histórico de Puebla (tesis doctoral). Barcelona, España: Universitat de Barcelona.

- (2013). Un proyecto de espacio público y participación ciudadana: Resumen el Barrio de San Antonio y Santa Anita. Revista Iberoamericana para la Investigación y el Desarrollo Educativo., 1.

- Atanacio Medellín, I. (2013). Estrategia Programa Integral Urbano Social: La inclusión social como herramienta de acción y planeación en la ciudad de Puebla. CIUMA.

- De La Torre Sánchez, C. E., Morales Flores, P., Aco Castañeda , B., Bautista Hernández, M., \& Rojas Salgado, C. J. (2013). Estudio comparativo en procesos participativos para la regeneración de tres barrios del Centro Histórico de la ciudad de Puebla.WATERFR@ NTS, 28, 78-96. Recuperado de http://www.raco.cat/index.php/ Waterfront/article/view/272772/360

Leicht, H. (1967). Las calles de Puebla: estudio histórico (Segunda edición, Séptima reimpresión ed.). Puebla, México: Gobierno del Estado de Puebla. Comisión de Producción Cultural. 
Peralta, V. M. (1998). Vecindad, intimidad y fusión de reciprocidades. Plaza y Valdés - BUAP.

Querol, M. Á. (2010). Manual de Gestión del Patrimonio Cultural (Primera edición ed.). Madrid, España: Akal.

Valera, S. y Pol, E. (2001). El concepto de identidad social urbana: una aproximación entre la psicologia social y la psicología ambiental. Psicología Ambiental, Programa del Doctorado Espacio Público y Regeneración Urbana. Arte, teoría y conservación del patrimonio. Recuperado el 6 de Junio de 2014, de http://www.ub.edu/escult/ doctorat/html/lecturas/identidad.pdf

UnesCo (2016). La UnesCo por las ciudades sostenibles. Obtenido de http:// es.unesco.org/sustainable-cities

Vélez Pliego, F. M., Guzmán Álvarez, A., et Al. (1997). Cartografía histórica de Puebla. Puebla. 